\title{
BIM: A Methodology to Transform Business Processes into Software Systems
}

\author{
Francisco J. Duarte ${ }^{1}$, Ricardo J. Machado ${ }^{1}$, and João M. Fernandes ${ }^{2}$ \\ 1 Departamento de Sistemas de Informação, Universidade do Minho, Portugal \\ 2 Departamento de Informática, Universidade do Minho, Portugal
}

\begin{abstract}
This manuscript proposes a guiding methodology to obtain a software system that supports the execution of the business processes existing within an organization. The methodology promotes the usage of business process reference models and intends to reduce the implementation time of the software systems. The methodology assumes four distinct phases and several abstraction levels and is applicable both when developing systems from scratch or in re-engineering contexts. The methodology embodies a special phase to handle the diversity of the business processes of an organization. By tailoring process reference models and by considering the characteristics of a specific organization, a proper set of business processes is derived for that organization. Then, we can obtain a suitable information system and implement its automatable parts in a software solution that can run on top of open source software frameworks. We also present four new supporting concepts to the methodology, and a summarized execution of it.
\end{abstract}

Keywords: BPM, BIM, business implementation methodology, software, software development process, OSS, COTS, EPF.

\section{Introduction}

For business companies, representing the real world business processes in software systems is an important issue during their development and utilization. Currently, the major software houses provide solid solutions to support in software systems the execution of business processes, usually with Commercial OffThe-Shelf (COTS) systems, like Enterprise Resource Planning (ERP), Customer Relationship Management (CRM), and Supply Chain Management (SCM). The technologies used inside those COTS systems are hidden from the users, and, in the best cases, standard interface technologies (e.g., web services) are available to interact with them. In addition, when the set of business processes implemented inside the COTS system is not sufficient to support the operation of a company, a significant effort to extend the implemented processes is required, either in new projects or in re-engineering contexts. The customization of a given COTS system requires a deep knowledge on the specific options it offers. Additionally, proficiency in very specific programming languages (e.g., ABAP in SAP R/3) or in the COTS specific business model and interfaces is usually demanded in 
order to support the scripting inside the COTS system or to execute a proper external use of interfaces.

To deploy COTS systems, some methodologies are proposed by software vendors or are developed by consulting companies that implement those systems. These methodologies focus on tailoring the COTS system to a specific client, and in most cases assuming that the current business operations of the client should not be deeply challenged. Thus, during a COTS implementation project, the client organization cannot be sure that the most appropriate set of business processes is implemented in the software solution, neither that the COTS implemented business processes follow world-class reference business process models. Additionally, there is no guarantee that the effort needed to upgrade a subset of the implemented business processes is manageable for the organization.

Typically, the supporting tools for a COTS system load, unload, extend, or customize business processes, but only focusing on implementation issues, rather than having a business-oriented perspective. Some platforms, like ARIS [1], provide a basis for editing the software system that implements business processes, usually following proprietary process reference models. Generically, business process implementation methodologies present the following problems:

1. Methodologies are not holistic, because that they do not always start with a process reference model and they do not always end up in implemented software;

2. Methodologies are not directed neither to use mixed vendor software components nor to use Open-source Software (OSS);

3. The time to implement a software system based on non-executable business process descriptions is long;

4. There is a lack of automation for model transformation activities;

5. It is hard to pick a preferred process reference model and use it inside an established methodology;

6. Many methodologies are proprietary and induce activities caused by the available COTS software characteristics;

7. A clear focus on the quality of the business processes content is missing. Instead, a focus on the target COTS software is observed.

According to [2], the objectives of modeling a software process are to facilitate human understanding and communication, to support process improvement and management, to provide automated guidance in performing the development process, and to automate execution support. Proposals exist, like [3], to increase the automation degree in model transformations from UML into BPEL, to mechanize BPM models [4], or to use XML to allow automation of dynamic business processes [5], but a holistic approach to transform business requirements into running software is still missing. In [6], Enterprise Service Buses (ESB) are considered as a state of the art solution for a capable and manageable integration infrastructure for web services and Service-oriented Architectures (SOA). ESBs implement a message backbone an based on open-standards designed to enable the implementation, deployment, and management of SOA-based solutions. We propose a methodology called Business Implementation Methodology (BIM) to 
better handle the above enumerated problems, to handle properties for processes suggested in [2], and to use state of the art software frameworks. BIM starts with generic business process reference models and ends up with running software. BIM is not attached to any proprietary software platform neither to specific notations for business processes or other process artifacts. Nevertheless, for the sake of reducing the project time, BIM encourages the usage of executable business process languages and techniques to increase the degree of automation in model transformations (e.g. 4SRS [7]). BIM also provides activities to choose and use reference models and customize them to a specific organization. BIM incorporates the idea that the quality of a business software is better if, at the proper time, quality business processes are designed and presented to the software development team. BIM also promotes the usage of OSS and software provided by different vendors.

The following are the main objectives of BIM:

- To provide a holistic approach to guide software implementations for process oriented organizations, following state of the art process reference models;

- To cope with different and mixed software technologies to support the operation of real world business processes.

In the next section, we describe related work on how to use generic process reference models inside business process organizations and we make some considerations regarding established COTS implementation processes. In Section 3, some concepts to support BIM are introduced. In Section 4, the proposed business implementation methodology is described in detail, namely its phases and transitions, its process, and the notations it supports. In Section 5, we present a running example to show the use of BIM, as well as some potential implementations of the proposed concepts. Finally, in Section 6, conclusions are presented and the future work is proposed.

\section{Related Work}

The goal of a methodology is to encourage an approach to solve a particular problem with a set of methods and techniques previously chosen [8]. A methodology can be presented as a series of phases, with associated techniques and notations [9].

The concept of process reference models arose from the idea of best practices shared among organizations. Reference models represent the business operations and internal structure of an organization. Reference models can be thought of as templates from which process models may be developed [10]. Consequently, if standard processes are to be used, standard roles to intervene with those processes can also be depicted in advance. Currently, generic business process reference models are available for organizations, like the Supply-Chain Operations Reference-model (SCOR) [11], the Information Technology Infrastructure Library (ITIL) [12], or the Enhanced Telecommunications Operations Map 
(eTOM) [13]. Business process reference models are standards accepted by organizations on how business processes can be properly designed and managed. Reference models are usually accompanied with recommendations, training events, books, and certifications, all providing a set of tools for implementation teams. This approach relies heavily on the human skills of the implementing team and it is not immune to project environmental conditions, like a low budget for training. After the adoption of a new set of business processes comes the need to implement most of them, quickly and with quality, in some software system. Some methodologies exist to help on the development of software systems for supporting business activities. Generic software development processes can include disciplines, like the Business Modeling discipline in the Rational Unified Process (RUP) [14], to help software developers understand and define the business context of their client in a set of process deliverables. In some of those models, notation is defined applying extensions to the Unified Modeling Language (UML) [15] to design business processes. The Business Modeling discipline provides key inputs to requirements and software design activities, namely a basis to decide which business processes will be implemented in software, or some business properties that will influence decisions related with non-functional requirements. COTS software vendors also propose methodologies, like Oracle PeopleSoft Enterprise - Rapid Start [16], Microsoft Business Solutions - Navision Rapid Implementation [17], or SAP AcceleratedSAP [18], that focus on the proper implementation of their own COTS technology, assuming that they will satisfy the clients' needs. Just considering the names of the presented methodologies, one can realize the importance of the time needed to implement a COTS system. For that reason, automation efforts, like deriving executable business processes [19], are crucial to help achieving a reduced implementation time. A complementing approach is to use iterations to provide the most needed parts of the solution as soon as possible, complemented with later additions. Also, efforts from non-profit consortia, like OASIS Business Centric Methodology [20], are focusing on the proper implementation of business processes with web services to provide ubiquitous connections among information systems and the people who use them. Considering the above mentioned reasons, it is advisable that a methodology to develop software systems supporting some set of business processes includes:

- a way to deal with standard process reference models;

- the integration of tailored business processes, as demanded by the client organization;

- the promotion of automation for its techniques;

- the capacity to obtain a quality software solution in a fast way.

As processes are becoming more and more automated, the management of processes will become automated as well [10]. In the near future, organizations must reach an internal level of maturity to allow focusing on building and improving excellent business processes, rather than focusing on the daily running problems of their processes and respective software implementations. 


\section{Proposed New Concepts}

BIM introduces four new concepts to support the use of process reference models during software development projects. The first concept, called Instantaneously Available Organization (IAvO), proposes the creation of templates for detailed business processes and roles, that afterward can be used by business entrepreneurs. This proposal extends the concept of process reference model. The second concept, designated Organizational Aspect (OA), is related with orthogonal characteristics that an organization can exhibit. For instance, an organization may want to implement some quality standard that is cross-cutting to all business processes. The third one, Process Framework (PF), is the main deliverable of BIM. During the BIM life-cycle, the PF is expected to pass through a series of states. The fourth concept, Orchestrated Business Object (OBO), is the software implementation of a business entity and its associated functionalities and data, compliant with business process reference models.

\subsection{Instantaneously Available Organizations}

Everyday, new organizations are created. Everyday, entrepreneurs think they have discovered a killer product or service that will bring a huge advantage over their competitors, either because the forthcoming product is an absolute premier, or because it incorporates new features that will overwhelm the competition. This business creation willing can be truncated with organizational problems. For the entrepreneurs, the internal configuration of their own organizations as well as the interfaces with suppliers and customers, can present difficult obstacles, either because they are not properly defined or, even worst, because entrepreneurs do not have any idea of what business content is necessary to be embodied into their own organizations. The set of common practices shared among organizations, operating inside the same business contexts, is not negligible. Furthermore, an organization from a vertical market can, and should, perform benchmarking activities to the processes of other organizations, either inside the same vertical market or not, and thus embodies processes from other organizations placed in a different vertical market. For that, it seems plausible that business practices materialized in business processes may be shared among organizations. For those reasons, we propose the concept of IAvO. The main goal behind using the IAvO concept is to have out-of-the-box organizations that entrepreneurs can pick and materialize on a concrete organization. This approach can also be used when a given organization wants to redesign itself. This concept assumes that entrepreneurs and organizations reach a high maturity level such that they are "humble" enough to use standard best practices instead of only imposing their ideas for the design of the processes. Apart from the process definitions, standard roles to run the processes can also be designed. Standard roles can help entrepreneurs seeing the amount and qualifications of the human capital to be hired. Despite the business competition environment, from an information systems perspective, business processes can be shared with competition. 


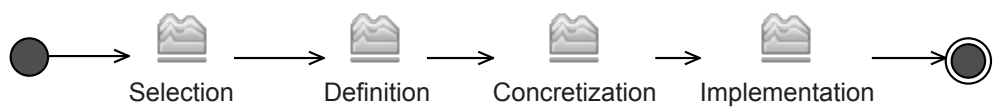

Fig. 1. BIM Delivery Process

This can happen because the main differentiation among competitors is based on products or core business specific activities (like a shop-floor layout), and mainly because the most decisive factor for the success of an organization is their own human capital. Inside BIM, IAvOs are considered in the first phase, where a selection of standard processes is made for later implementation. The $\mathrm{IAvO}$ concept differs from reference models because it is much more concrete. IAvOs share the same notations with reference models.

\subsection{Organizational Aspects}

An OA is a cross-cutting organizational concern. The concept of OA is inspired on the concept of Aspect, as introduced in Aspect-Oriented Programming (AOP) [21]. During the first BIM phase, we propose that the business analyst picks standard process reference models and, using one or more OAs, he/she creates an adequate process reference model for the organization under consideration. The process reference model incorporates a set of activities semantically compliant with some organizational standard. If some cross-cutting concern within the organization exists, then an OA can model it. With the OA concept, process reference models compliant with organizational standards, like European Foundation for Quality Management (EFQM) Excellence Model [22], can be designed or imported from the market. The OA extension mechanism, similar to that of the advices in AOP, can be a basis to use world-class added value processes without ignoring compliance with external business drivers. At the end, the surviving processes include all the desired characteristics of the chosen orthogonal quality standards. Of course, conflicts may arise, such as when creating a lean production process while maintaining traceability data, but they will be more easily tracked and explicitly solved.

Process reference models with OAs can also provide gains to organizations, when:

1. a new organization is to be created from scratch (e.g., a new subsidiary) assuring the correct transposition from mother organization of:

(a) the local processes that may be compliant with central processes;

(b) the standards requested locally and immediately fulfilled (e.g., social responsibility);

2. country-specific customization of processes (e.g., human resources) can be obtained from an $\mathrm{IAvO}$;

3. for an existing organization, during a Business Process Re-engineering, current processes can be compared with IAvO processes that are compliant with some standard. 
This last possibility provides an accurate visualization of the as-is and to-be states of the organization, and ensures that the organization can achieve the standard certification because a quantitative measure of the work is available.

\subsection{Process Framework}

A crucial concept in BIM is the PF, which is a set of process models and tools that allow the development project stakeholders to manage business processes at different states. A PF contains:

- a set of business process reference models;

- a set of allowed activities that can be performed on included process reference models;

- a set of actors that can perform activities;

- a defined state;

- a business ontology.

The PF is the main artifact being manipulated during the BIM process life cycle. To help handling the PF, visualization tools are desirable. These tools depict the $\mathrm{PF}$ at its different states and interpret the chosen business process description language.

\subsection{Orchestrated Business Objects}

OBOs are pieces of software that implement business entities inside some specific business ontology. They expose functionality and data to the softwareimplemented PF in order to allow the orchestration of business processes. Each OBO is a black-box and it should be interchangeable with other OBOs implementing the same business entity. It is highly beneficial to have a set of OBOs already available prior to the last BIM phase in order to reduce the project time and to reuse code. The concept of creating a project for developing software based on previously developed components is similar to the one proposed by the Software Factories [23] approach. The OBO concept addresses the need to have different pieces of software orchestrated to implement a specific business process, with the capacity to be interchangeable and without causing any disruption in the software system behavior.

\section{A Business Implementation Methodology}

The opportunity to generate, in an automatized way, a running software system improves the quality of the resulting product. This eliminates errors during manual model transformations and allows the developers to focus on the definitions of adequate content of business processes and software environment instead of focusing on the correctness of the models transformations. Automation also induces a reduction in the development time. 
The primary goal of BIM is to guide the development of business software, ensuring the adequate support for the set of the running business processes inside an organization.

We describe BIM using to the Eclipse Process Framework (EPF) [24]. BIM is composed of four discrete time consecutive phases (Figure 1). During the first phase, a Selection of process reference models, OAs, and IAvOs is made, followed by a Definition of the chosen business processes to a particular project. Next, the Concretization of the business processes into an information system occurs. At this phase, yet there is no software implementation, and the organization may have alternative business processes defined for the same area. In the last BIM phase, one or more software Implementations are made for a subset of business processes concretized in the information system. The need to have different software implementations comes, for instance, if the organization is relying on an ERP which has annual upgrades. During those upgrade periods, the organization can benefit from having an alternative software system to overcome the inactivity of the ERP. Each BIM phase ends with a quality assessment (QA) milestone, in order to evaluate the maturity of the deliverables. The QA produces a statement expressing if the project is ready to move into the next phase (or to terminate, in the last QA). Each BIM phase follows the pattern implemented for the Selection phase activities (Figure 2). As a result, each BIM phase is composed of one or more iterations followed by a QA. While the expected quality and maturity levels of the artifacts, deliverables, and outcomes are not reached, or when some project change or constraint occur, iterations can be run incrementally inside the same BIM phase.

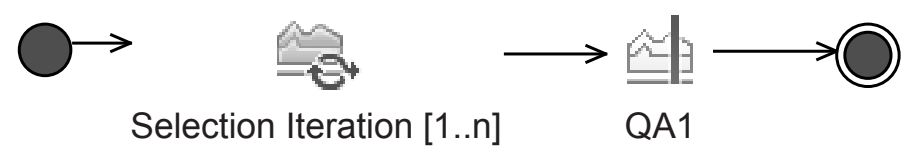

Fig. 2. BIM Selection Phase

During the development process, a set of state transitions must be carried on the PF, as shown in Figure 3. Starting with generic process reference models and IAvOs, an Instantiated PF is obtained. The business processes can be described, desirably in Business Process Execution Language (BPEL) [25], but also with Business Process Modeling Notation (BPMN) [26], UML, Event-driven Process Chains (EPC) [27], or Colored Petri Nets (CPN) [28]. The preference for BPEL comes from the easiness to execute it. The Instantiated PF has to consider the mission, vision, and strategic objectives, as well as other business constraints, of the organization. At this PF state, the description of the process-oriented organization is achieved, being the basis to derive the information system model. Afterward, when the PF is concretized into the information system, the PF is in the Runnable state because its processes, most likely not all, may be implemented in software. The bidirectional state transitions between the Runnable and the Software-implemented states are justified by the "loading" of the software system 
with a subset of the information system processes and by the "unloading" of some of those processes, becoming processes in a "vegetative" Runnable state. An ideal software implementation for the PF relies only in parametrization. There is also the possibility that some Generic PF can directly be moved into the Runnable PF state. This situation occurs when the organization does not want to make any customization of a generic process reference model or when the organization wants to fully adopt some IAvO. Desirably, the Runnable and the Softwareimplemented states represent the same amount of business content.

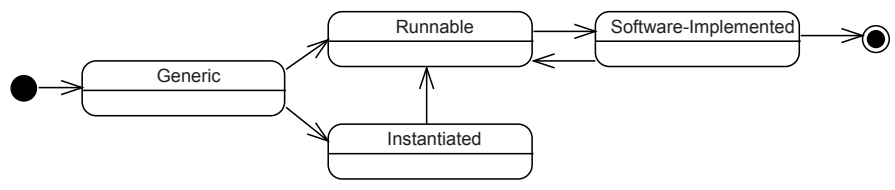

Fig. 3. PF States

BIM, generically summarized in Table 1, supports, during the first three phases, business process description languages. Because a software implementation is in its scope, during the last BIM phase there is a need for a computational model. In the Selection and Definition phases, activities are occurring in the domain of requirements. During the Concretization phase an information system for the organization is designed, with manual and software-implemented business processes. PF states are directly related with the BIM phases.

Table 1. BIM Summary

\begin{tabular}{l|ccc} 
BIM Phase & Metamodel & Domain & PF State \\
\hline 1. Selection & BPEL, BPMN, & Business & Generic \\
& EPC, CPN,$\ldots$ & Requirements & \\
\hline 2. Definition & BPEL, BPMN, & Business & Instantiated \\
& EPC, CPN,$\ldots$ & Requirements & \\
\hline 3. Concretization & BPEL, BPMN, & Information & Runnable \\
& EPC, CPN,$\ldots$ & System & \\
\hline 4. Implementation & Computational & Execution & Software- \\
& Model & & implemented \\
\hline
\end{tabular}

\subsection{Activities of the BIM Phases}

Inside each BIM phase, process-related functions are performed by process roles. For the Selection phase, we present the activities in Figure 4. The role Business Analyst chooses from the market an adequate process reference model (e.g., $\mathrm{SCOR}$ ). It is advisable that he/she considers if there is available an IAvO that addresses the business processes covered by the project. The Business Analyst and the Client are responsible for defining which OAs are to be included in the PF. 


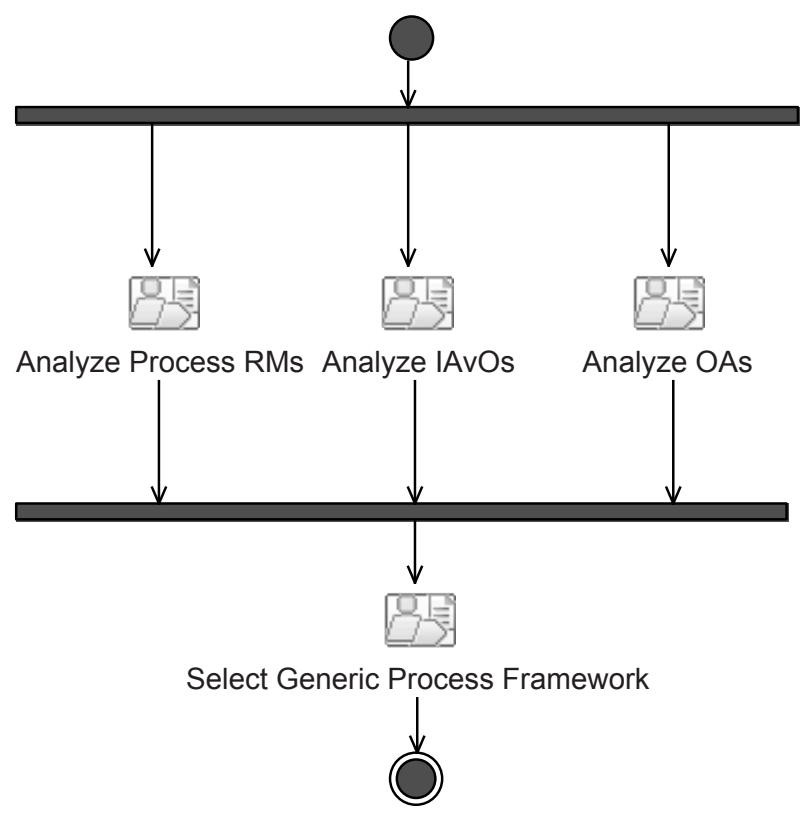

Fig. 4. Activities in BIM Selection Phase

The most important deliverable generated during the Selection phase is a mature and world-class generic process reference model chosen and ready to be used in the next phase. Besides the intrinsic business quality of the process reference model, its form of representation must also be chosen. Using standard process notation languages, mixed with informal graphical notation, or even using some text descriptions, can be accepted if they bring clarity to the description of the Generic process reference model that was chosen. The Generic reference model selected in the Selection phase is used to reach an Instantiated PF during the Definition phase. The Instantiated PF includes the client's view and future strategy. Business-Driven Development [29] can be used to create a proper alignment of the vision of the organization with IT tools. One key aspect of the Definition phase (see Picture 5) is the explicit utilization of an immaterial role represented by the Generic PF.

We propose that, even when the Client or the Business Process Architect do not explicitly consider world-class business processes, and for the sake of the organization, one must consider the demands of the Generic PF role in order to derive a proper Instantiated PF. The Client is not always able to decide the best solution for his/her own organization. This situation can be triggered by the ignorance of best-practices outside his/her own business environment or by the human representatives of the Client that do not always put the best interests of the organization on top of their own ones. 

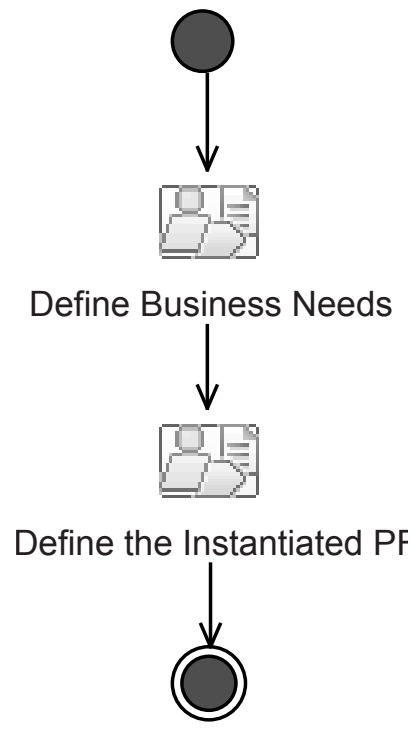

Fig. 5. Activities in BIM Definition Phase

After a BIM project, the complete information system, consisting both of software-implemented and manual processes, needs a regular audit activity. The $\mathrm{PF}$ in the Runnable state is vulnerable to end users that do not want to use the business processes of the organization, normally expressing it by creating personal worksheets and databases. Thus, we propose an audit to be carried on periodically (e.g. yearly), in order to check the compliance of the current Software-implemented PF with respect to the one designed during the BIM project. This check can only be applied if the business environment during BIM project time still applies, otherwise a new run of BIM is needed. This activity prevents changes in the Software-implemented PF without proper validations. During the audit, it is recommended to check if the manual processes are still executed the way they were planned.

Undesired changes in business process should not be confused with the ability to cope with continuous improvement activities. Whilst the former may turn the results of activities against their own host organization (e.g., the optimization of a production process that conducts to an increased product stock without a proper planning of the available space), the latter are mandatory in any organization that seeks business excellence. The goal of the third BIM phase, Concretization (see Figure 6), is the design of the Runnable PF, based on the Instantiated PF obtained in the Definition phase. The Business Process Architect role must include into the Runnable PF alternative designs for the same business processes, as well as the decisions about which business processes will have a software implementation. 


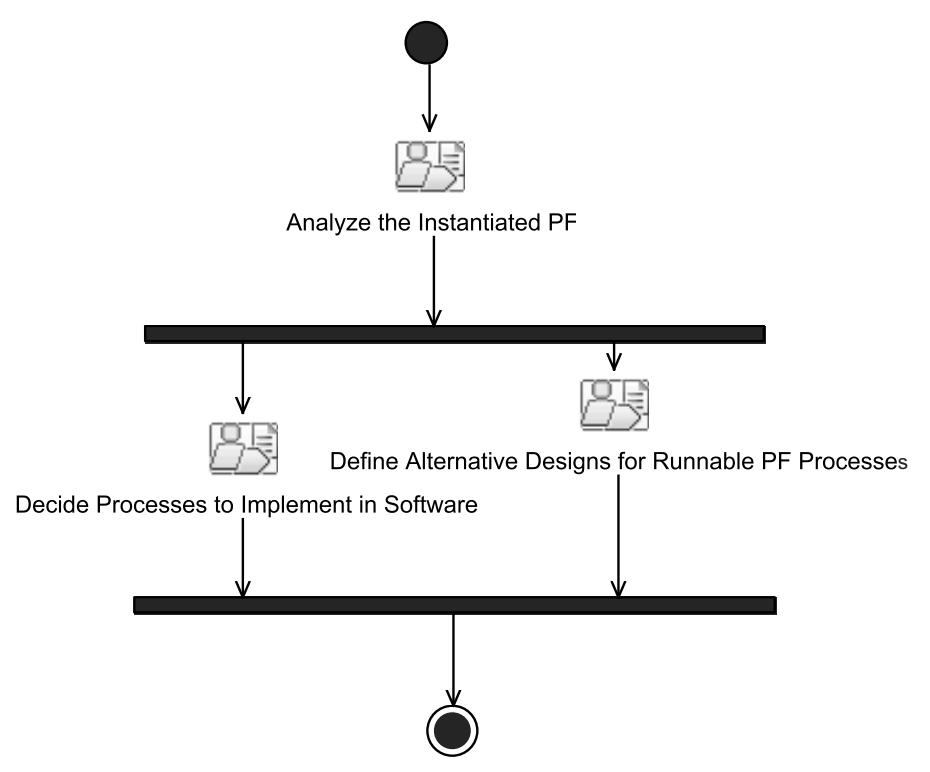

Fig. 6. Activities in BIM Concretization Phase

Finally, during the Implementation phase (Figure 7), the Runnable PF is implemented in software. Two distinct approaches can be taken to solve the problem of generating a software solution. The first is to generate code starting with previous defined models. The second is to use already developed code, customized to cope with the requirements. With the latter approach, mixed vendor pre-developed software can be used, if compliant with the requirements of the Runnable PF. In this scenario, the client would not be tied to a particular COTS software vendor, neither he would have to rely on web services for which a proper service level agreement is unfeasible. We believe that having mixed vendor software to support business operations inside an organization brings benefits for the quality of the implemented solution, because the client can have the ability to load and unload software implementations for a part or for a complete business process. During the BIM Implementation phase, the designer of the behavior of the Software-implemented PF is the Business Process Architect and not the Software Engineer. The latter role is only concerned with providing a running software infrastructure for business processes to run. The existence of pre-developed software (OBOs) indicates that additional (outside the BIM project) software development activities may be needed and accomplished under the responsibility of the Software Engineer.

The Software-implemented PF is customizable and is the cornerstone to compare the complete OBO functionality with the Runnable PF requirements. Conflicts between the Software-implemented PF and the Runnable PF can be detected, when the latter requires more functionalities than the ones available in 


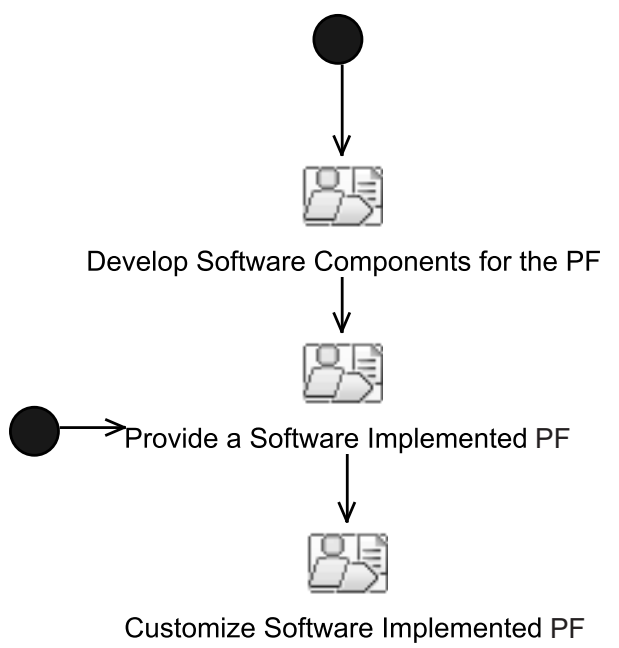

Fig. 7. Activities in BIM Implementation Phase

the OBOs. This type of conflicts may demand the acquisition or the development of more OBOs, or extension of the existing ones.

\subsection{Supporting Technology}

In this section, we present a possible implementation for the Software-implemented PF, based on free software. It uses the Apache ServiceMix 4 (SMX) [30], a wellknown ESB. Within an organization, a significant number of applications and platforms usually exist. This diversity requires different data formats and communication protocols to be integrated. In recent years, several technologies, such as Enterprise Application Integration (EAI), Business-to-Business (B2B), Service Oriented Architecture (SOA), and Web Services, were proposed to solve these problems. These technologies address some of the integration problems, but they are proprietary, expensive, and time-consuming to implement [31]. The ESB approach provides distributed messaging, routing, business process orchestration, transactions, reliability and security. SMX is a standard-based ESB since its design is compliant with the Java Business Integration (JBI) specification [32] and it is based on OSGi service platform [33] architecture. Since SMX is widely accepted, open source, and based on open standards, it can provide a quality, flexible, and low cost solution to implement the PF. In SMX, software components are added as OSGi bundles, like the Apache Orchestration Director Engine (ODE) [34]. ODE is a BPEL executing engine.

SMX, ODE, and BPEL, overcome the need for model transformations in the $\mathrm{PF}$, since the same BPEL model can be used in all of the BIM phases. Therefore, BPEL can be used to describe the Generic, the Instantiated, the Runnable, and the Software-implemented PFs. During the BIM life-cycle desirably only the business semantics needs to be changed, maintaining the same language syntax. 
Thus, one can describe, or pick from the repository of the Runnable PF, a business process in BPEL and drop it on the ODE within SMX, allowing the PF to change from the Runnable state to the Software-implemented state. In this scenario, OBOs are OSGi bundles and OBOs orchestrations are the configurations deployed into the ODE. These two implementations define a Domain Specific Language (DSL) [35] in the domain of the adopted process reference model. It means that the less abstract parts of the processes of the Generic PF must also exist in the SMX as bundles (Section 5 presents an example). During a BIM project it is assumed that all the needed OBOs (derived from the DSL of the Generic PF) are implemented as bundles in the SMX. If this is not the case, then one must acquire the needed OBOs or initiate a project to create them.

\section{A Summarized Execution of the BIM}

To demonstrate BIM, we use BPEL and SCOR. SCOR is configurable and contains descriptions and relationships among processes, as well as standard metrics to evaluate process performance. SCOR also embodies management practices and methods to reach some of its deliverables (e.g., Business Scope Diagrams) [11]. The three most abstract levels of business processes in SCOR are:

- Level 1: Top Level (Process Types). There are only five process types (Plan, Source, Make, Deliver, Return);

- Level 2: Configuration Level (Process Categories). Organizations implement their strategy by choosing different configurations to their process, like "MakeTo-Order" or "Make-To-Stock";

- Level 3: Process Element Level (Decompose Processes). Organizations finetune their strategy by creating process element definitions, state their inputs, outputs, metrics, and pointing best practices.

In SCOR, the levels below the third one are left to organizations to implement their own specific practices so that they can cope with changing business conditions and achieve competitive advantages. In these lower levels, organizations are invited to use classic hierarchical process decomposition:

- Level 4 contains "Tasks" to implement "Process Elements";

- Level 5 contains "Activities" to implement "Tasks";

- Level 6 is where detailed actions are used to implement "Activities".

As an example, we use level 3 "D1.10 Pack Product" Process Element. It is part of the Configuration Level "Deliver Stocked Product" (level 2), and of the Top Level "Deliver" (level 1). The Tasks shown in Figure 8 represent a chosen configuration for the Process Element "D1.10 Pack Product". Each Task, like "D1.10.1 Sort Product", can be refined with another BPEL diagram.

In our example, the level 3 of SCOR will define the DSL used in all BIM phases. This means that the OBOs are all SCOR level 3 Process Elements and that they must exist inside the SMX as bundles. The internal architecture of each OBO is left open. BPEL is built with a Web Services mindset, which could 
prevent the mapping of manual processes during all the BIM phases. To overcome this limitation, all the process components, either with a manual or software existence, are mapped in the BPEL description. When a manual action is to be executed, its trigger comes from the software system and the result of its execution must also be informed to the software system.

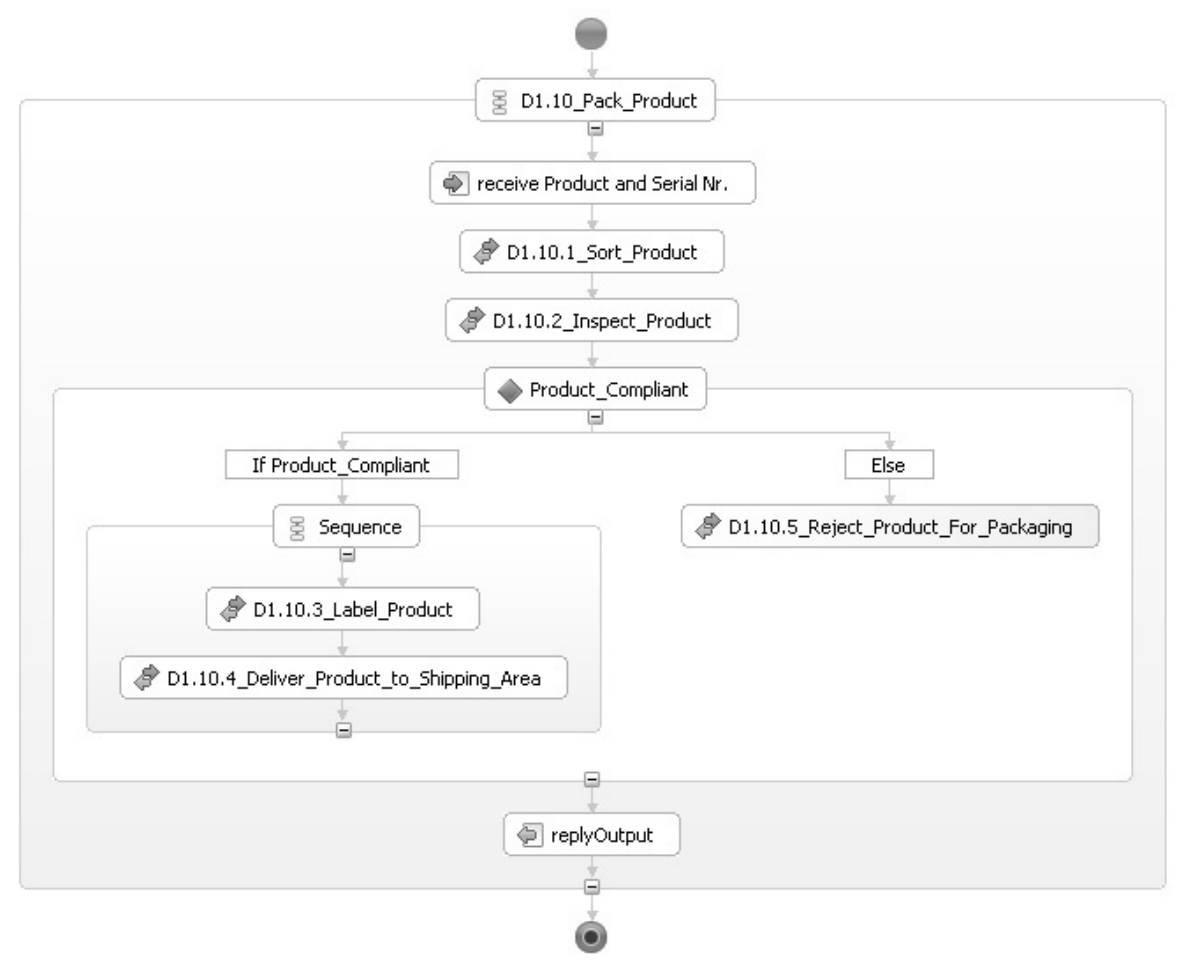

Fig. 8. SCOR Process Element D1.10 described in BPEL

A set of configurations of SCOR Process Elements can materialize an IAvO. After defining the Configuration Level for Top Level processes and define the appropriate Process Elements, a Business Architect can also define all Tasks, Activities, and detailed actions, thus creating an IAvO.

The degree of variability inside the IAvO is an option of its creator. It can be tracked by alternative definitions contained inside the same level of SCOR for the same process component, i.e. an IAvO can allow its users to employ "Make-ToStock" or "Make- To-Order" strategies. If the IAvO creator wants to completely define the organization, he/she can create all the six levels of details. Depending on the business context of a process component, namely the number of goods or the time to handle them in packaging, a proper set of business roles can be drawn and incorporated into the IAvO. For instance, the IAvO's business process described in Figure 8 implies that four business roles may exist: Product Sorter, 
Product Inspector, Product Labeler, and Product Deliverer. The IAvO creator can define if the business roles are to be performed by the same individual or by a set of persons. Afterward, the IAvO user can decide if the IAvO will be implemented exactly as it is defined (e.g., when a multinational organization is creating twin companies) or if some deviation is possible (e.g., when he/she is an independent entrepreneur).

To show an OA, in our SCOR-based IAvO, we consider the implementation of an Excellence Model in our organization, like the EFQM model [22]. In the EFQM model, an important part of the business activities is their reviewing. Inside an organization, one must know how well the business activities were performed, what are the causes for not achieving expected results, and how our business activities can be improved based on some measure or benchmarking. Such business concerns may be expressed at different levels of the SCOR model: if we are "lazy", we may review our processes at Process Type level; if we are "micro-managers", we may review all our level 6 detailed actions. We suggest to implement OAs at SCOR level 3, because it is the first level where concrete activities are performed. Additionally, this level is sufficient abstract and manageable to cope with changes in its Tasks, Activities, and detailed actions. For that to occur, one can decide to perform a review, every time the business process is executed. This additional Activity, the OA, can also be implemented as a bundle. The interception of the end of the Process Element D1.10 Pack Product can be done, inside the SMX, by a route in Apache Camel [36], a Spring based integration framework that implements Enterprise Integration Patterns [37].

Following, we present a summarized execution of the BIM for a goods manufacturer. In the Selection phase, the execution of the activities (see Figure 4) resulted in the the selection of the SCOR, an OA to allow reviewing all the process elements, and no IAvO considered. With these results, we selected as our Generic PF the SCOR. At this stage, a representation in BPEL of the reference model (SCOR in the case) must be available. The QA 1 is performed (see Figure 2 ) and the result is that the SCOR, due to its characteristics and nature, is approved as an adequate reference model.

For the Definition phase (see Figure 5), the execution of activities resulted in the identification of Quality as a major business need. The SCOR was extended by defining Tasks, Activities, and detailed actions. One extension example is showed in Figure 8 where, and resulting from the execution of D1, was included a Task "D1.10.2 Inspect Product" to guarantee high quality levels in product deliveries. The Generic PF role was instantiated with SCOR, meaning that the Instantiated PF explicitly considered it. After this second BIM phase, it is required to have the new Instantiated PF represented in BPEL. The Instantiated $\mathrm{PF}$ is evaluated in the QA 2.

In the Concretization phase (see Figure 6), activities execution resulted in the identification of a need for an alternative "D1.10 Pack Product" Process Element to allow the movement of products to shipping areas when no labels are present. Both alternative Process Elements "D1.10 Pack Product" will have software implementations. Due to the alternative "D1.10 Pack Product", the 
Tasks "D1.10.3 Label Product" and "D1.10.4 Deliver Product to Shipping Area" (in Figure 8) will exchange their positions for the alternative design (not showed). Then QA 3 is performed.

For the BIM Implementation phase (see Figure 7), activities resulted in the customization of ODE by deploying the BPEL process definitions made for the Runnable PF. The needed "D1.10 Pack Product" OBOs are loaded into SMX. BIM ends with the the execution of QA4 to prove that there is an Softwareimplemented PF in the SMX running according the organization requirements.

To properly execute BIM some preconditions are needed. The most important is that the client organization is willing to improve its business processes by considering external inputs, namely in the form of process reference models. It is also critical to have human resources able to understand and use effectively process reference models, and to have skilled IT staff able to use different software frameworks. During the project phase, the project team members must have the necessary empowerment and management support to transform the business processes.

From the few executions of BIM, the main feedback from business process experts is that the usage of reference models is time consuming at the beginning due to the used notations and to the capacity needed to understand a reference model. Software experts point out that having requirements expressed by customized reference models in a clear form and early in the project increases the quality of the resulting software and shortens the time needed to implement it, mainly by avoiding cycles during the specification and validation of requirements.

\section{Conclusions and Future Work}

This manuscript presents BIM, a high-level methodology to properly implement in software a desired set of business processes. At present, BIM is designed to act as a guide using a phased development process. Also, the explicit inclusion of new concepts, like IAvO, OA, PF, and OBO, can spot the importance of the underlying activities and trigger their discussion. IAvOs allow entrepreneurs, namely the inexperienced ones, to start a business with a higher probability of success, and/or to reduce the time needed to set up the internal structure of an organization, by defining a core set of business processes and business roles. OAs can help on configuring business architectures to cope with cross-cutting business concerns, like quality standards, without significant effort. PFs can be the basis to smoothly transpose requirements and definitions from the business into the software execution domain. OBOs constitute an important concept, namely by allowing the client organization to use a mixed vendor software solutions to support its business processes. BIM can also help on considering process reference models during business process implementation projects, by embodying bestpractices and thus improving business operations.

BIM is not attached to any software implementation. BIM allows the usage of different vendor software, including OSS, according to the best interest of the client and not necessarily the best interest of the software vendor. BIM promotes 
the usage of COTS and OSS by using already developed software frameworks, like Apache ServiceMix, namely during the Implementation phase.

Organizations must be immune to egocentric employees with "fantastic" ideas. Sometimes, proposals coming from world class processes are offered but not considered due to the pride of those employees. This behavior can be a serious obstacle to improve the internal structure and the results of the organization. BIM explicitly considers business process reference models to wide the mindset of organizations, but also considers that detailed refinements of business processes are the way for organizations to express their excellence.

BIM is a wide-scope methodology, since it deals with business definitions and also with software implementations. For that, BIM is currently proposed in a high-level of abstraction, so that it can consider several concepts of the domains it covers and it can remain independent of technologies, notations, and methods. BIM brings four main advantages over current methodologies:

- it has a holistic scope, since it starts from generic process reference models and ends in implemented software systems;

- it avoids model transformations by using the same model for business processes during all the life-cycle;

- it promotes that a software system can include components from distinct software vendors, by using the best OBOs from each vendor to compose a valuable solution for the client organization;

- it can significantly reduce the time to implement a solution, since the very same model to describe and execute business processes is used along the complete life-cycle of BIM.

BIM is adaptable to different process reference models and software solutions, it addresses both the business and the software domains with a traceable artifact, the PF, and it proposes automation.

In the future, several issues will be tackled to improve BIM: (1) develop tools to support it; (2) extend the guidance to properly tailor the methodology to a specific project taking into consideration the possible methods and techniques that can be used during a BIM project, the skills of the development team, the business context of the organization, the business requirements for the process framework, or the available implementation time; (3) to test BIM in demanding business projects; (4) to develop IAvOs, using some business process description language.

\section{References}

1. Scheer, A.: ARIS-Business Process Modeling. Springer-Verlag New York, Inc., Secaucus (2000)

2. Curtis, B., Kellner, M., Over, J.: Process modeling. Communications of the ACM 35(9), 75-90 (1992)

3. Gardner, T.: Uml modelling of automated business processes with a mapping to bpel4ws. In: Proceedings of the First European Workshop on Object Orientation and Web Services at ECOOP 2003, Citeseer (2003) 
4. Hepp, M., Leymann, F., Domingue, J., Wahler, A., Fensel, D.: Semantic business process management: A vision towards using semantic web services for business process management. In: IEEE International Conference on e-Business Engineering, ICEBE 2005, pp. 535-540. IEEE (2005)

5. Chen, S., Chung, J., Cohen, M., Fu, S., Gottemukkala, V.: Dynamic business process automation system using xml documents, uS Patent 6,507,856, January14 (2003)

6. Papazoglou, M., Traverso, P., Dustdar, S., Leymann, F.: Service-oriented computing: State of the art and research challenges. Computer 40(11), 38-45 (2007)

7. Machado, R., Fernandes, J., Monteiro, P., Rodrigues, H.: Transformation of UML Models for Service-Oriented Software Architectures. In: The 12th IEEE International Conference on the Engineering of Computer-Based Systems (ECBS 2005), Greenbelt, Maryland, USA, pp. 173-182 (2005)

8. Ghezzi, C., Jazayeri, M., Mandrioli, D.: Fundamentals of Software Engineering (1991)

9. Rumbaugh, J., Blaha, M., Premerlani, W., Eddy, F., Lorensen, W.: Object-oriented modeling and design. Prentice-Hall, Inc., Upper Saddle River (1991)

10. Snabe, J., Rosenberg, A., Møller, C., Scavillo, M.: Business Process Management: The SAP Roadmap. Galileo Press, SAP Press (2008)

11. Supply-Chain Council, Supply Chain Operations Reference Model (SCOR), Version $9.0(2008)$

12. OGC, Best management practice: Itil v3 and iso/iec 20000 (2008)

13. Kelly, M.: The telemanagement forum's enhanced telecom operations map (eTOM). Journal of Network and Systems Management 11(1), 109-119 (2003)

14. Kruchten, P.: The rational unified process: an introduction. Addison-Wesley Longman Publishing Co., Inc., Boston (2000)

15. Rumbaugh, J., Jacobson, I., Booch, G.: The Unified Modeling Language reference manual. Addison-Wesley Longman Ltd., Essex (1998)

16. Oracle PeopleSoft, Peoplesoft enterprise - rapidstart for healthcare (2007),

http://www .oracle.com/industries/healthcare/

oracle-healthcare-enterprise-rapid-start-ds.pdf

17. Microsoft Navision, Automotive manufacturer deploys integrated erp solution and boosts customer service in just 15 days (May 2005)

18. Daneva, M.: Six Degrees of Success or Failure in ERP Requirements Engineering: Experiences with the ASAP Process. In: International Workshop on COTS and Product Software: Why Requirements are so Important, vol. 11 (2003)

19. Weber, I., Haller, J., Mulle, J.: Automated derivation of executable business processes from choreographies in virtual organisations. International Journal of Business Process Integration and Management 3(2), 85-95 (2008)

20. OASIS, Business-Centric Methodology (BCM), OASIS Std., Rev. 1.0 (May 2006), http://www . oasis-open.org/committees/download.php/17942/

BCM. OASIS.Specification.2006-05-01.zip

21. Kiczales, G., Lamping, J., Mendhekar, A., Maeda, C., Lopes, C., Longtier, J., Irwin, J.: Aspect-Oriented Programming. In: Aksit, M., Auletta, V. (eds.) ECOOP 1997. LNCS, vol. 1241, pp. 220-242. Springer, Heidelberg (1997)

22. EFQM, EFQM Excellence Model - Large Companies, Operational and Business Units version. European Foundation for Quality Management (2001), http://www.efqm. org

23. Greenfield, J., Short, K.: Software Factories: Assembling Applications with Patterns, Models, Frameworks, and Tools. Wiley (2004) 
24. Eclipse EPF project. Eclipse process framework (epf). Eclipse Process Framework (EPF) project (January 2011), http://www.eclipse.org/epf/general/description.php

25. OASIS, Web Services Business Process Execution Language v2.0, OASIS Std., Rev. 2.0 (April 2007), http://docs.oasis-open.org/wsbpel/2.0/0S/wsbpel-v2.0-0S.pdf

26. White, S.: Introduction to BPMN, IBM Cooperation, pp. 2008-2029 (2004)

27. Scheer, A., Thomas, O., Adam, O.: Process Modeling Using Event-driven Process Chains. In: Process-aware Information Systems: Bridging People and Software through Process Technology, pp. 119-145. Wiley, Hoboken (2005)

28. van der Aalst, W.: Challenges in business process management: Verification of business processes using Petri nets. Bulletin of the EATCS 80, 174-199 (2003)

29. Mitra, T.: Business-driven development, IBM Developer Works (2005), http://www.ibm.com/developerworks/webservices/library/ws-bdd

30. A. S. Foundation. Apache servicemix 4.3. Apache Software Foundation (March 2011), http://servicemix.apache.org

31. A. S. Community, Apache servicemix 3.x users' guide, web page (February 2008), http://servicemix.apache.org/users-guide.html

32. Ten-Hove, R., Walker, P.: Java Business Integration (JBI) 1.0-JSR 208 Final Release, Sun Microsystems, Inc. Std. (2005), http://jcp.org/en/jsr/detail?id=208.

33. T. O. Alliance, OSGi Service Platform Core Specification 4.2, The OSGi Alliance Std. 4, Rev. 4.2 (June 2009), www.osgi.org

34. Apache Software Foundation, Apache ODE User Guide, Apache Software Foundation (2009), http://ode.apache.org/user-guide.html

35. van Deursen, A., Visser, J.: Domain-specific languages: An annotated bibliography. ACM Sigplan Notices 35(6), 26-36 (2000)

36. Apache Software Foundation, Apache Camel Manual, 2nd edn, Apache Software Foundation (2009), http://camel .apache.org/manual/camel-manual-2.0-SNAPSHOT .pdf

37. Fowler, M.: Patterns of enterprise application architecture. Addison-Wesley Professional (2003) 